\section{SMGrøup}

\section{SM}

\section{Musculoskeletal Disorders}

\author{
Article Information \\ Received date: May 14, 2018 \\ Accepted date: Jun 04, 2018 \\ Published date: Jun 07, 2018

\section{*Corresponding author}

Wangdo Kim, School of Mechanical and Aerospace Engineering, Seoul National University, Seoul Korea,

Tel: +82-010-8644-2145;

Email: mwdkim@gmail.com

Distributed under Creative Commons CC-BY 4.0

Keywords Useful sensitivity; Knee instantaneous axis; the haptic control; Knee tensegrity system

Abbreviations KIA: knee instantaneous axis; GRF: Ground reaction force; COP: The Center of pressure

Article DOI 10.36876/smmd.1027

\title{
Rules for the Haptic Control of Locomotion
}

\author{
Wangdo Kim* \\ School of Mechanical and Aerospace Engineering, Seoul National University, Seoul Korea
}

\section{Abstract}

Perceptual psychology suggested that behavior was controlled by information about the world and the self conjointly. The information has now been described. What about the control? In this article, we asserted that locomotion was controlled by rules. Surely, however, they are not rules enforced by an authority. The rules are not commands from a brain; they emerge from the human-environment system.

We found that the rule is: The line of the Ground Reaction Force (GRF) vector is very close to the Knee Instantaneous Axis (KIA). It aligns the knee joint with the GRF such that the reaction forces are torqueless. The reaction to the GRF will then be carried by the whole structures on the body instead.

\section{Introduction}

'The haptic control does not have a satisfactory theoretical basis,' wrote psychologist James J. Gibson three decades ago. He then asked 'Can an ecological approach to the psychology of perception and behavior provide it [1]?' Clearly, his opinion was yes and we agree. In this article, we expand upon this idea by applying Gibson's concept of haptic control to the domain of locomotion in general and in particular to the design of braces and orthosis for musculoskeletal disorders [2].

The haptic system with its subordinate and superordinate systems constitutes a very elaborate perceptual apparatus. A great variety of perceptions can be achieved by it. But a systematic description of all these perceptions does not exist. Most experimenters on the sense of touch have concentrated on the sensations in controlled isolation, assuming that these were the components of perception. The question involves the perceiving of the general layout of environmental surfaces [3]. How is the arrangement or shape of these surfaces detected? The question is related to that of so-called space perception. The study of useful sensitivity, as distinguished from theoretically basic sensitivities, is only just beginning [4].

The question to be answered is this: How does a perceiver feel what he is touching instead of the cutaneous impressions and the bone postures as such? The objective of this study is to answer the question. In brief, the suggestion is that the joints yield geometrical information, that the skin yields contact information and that in certain invariant combinations they yield information specifying the layout of external surfaces.

\section{The Useful Dimension of Sensitivity of Touch to the Kinesthesis}

At anyone moment the orchestrated inputs from the shank $(\boldsymbol{S})$ and thigh $(\boldsymbol{T})$ segment specifies a set of bone directions relative to the knee and the direction of gravity. The bones and the extremities are thus linked to the environment (Figure 1). At anyone moment, the total input from the skin likewise specifies a pattern of contacts with touching surfaces, one of which is always the surface of a support. The skin is thus also connected to the environment by this simultaneous pattern.

The Arnold-Kennedy theorem of three axes [5] may be manifested when the two segment movements, $S$ and $T$, result in a third movement, the instantaneous axis of the knee, IAK, on cylindroids [6]. The IAK is defined by a linear combination of the two movements, $S$ and $T$ [5]. Then IAK must be that movement picked up among the many candidate movements on the cylindroids which are specially positioned to ground reaction force (GRP) [7]. Hence the ratio of the amplitudes about $\mathrm{S}$ and $\mathrm{T}$ is determined (Figure 1), which manifests that facts that the sensitivity of the knee joint to its orientation to both $\mathrm{S}$ and $\mathrm{T}$ of crucial importance for the elaboration of locomotion.

We define the sensitivity of touch to kinesthesis in accordance with the following terms:

A sensitivity $1=$ knee movement amplitudes divided by the amplitude of the movement of the foot $=\infty$.

A sensitivity $2=$ knee joint torque intensities divided by the intensity of the foot force $=0$. 


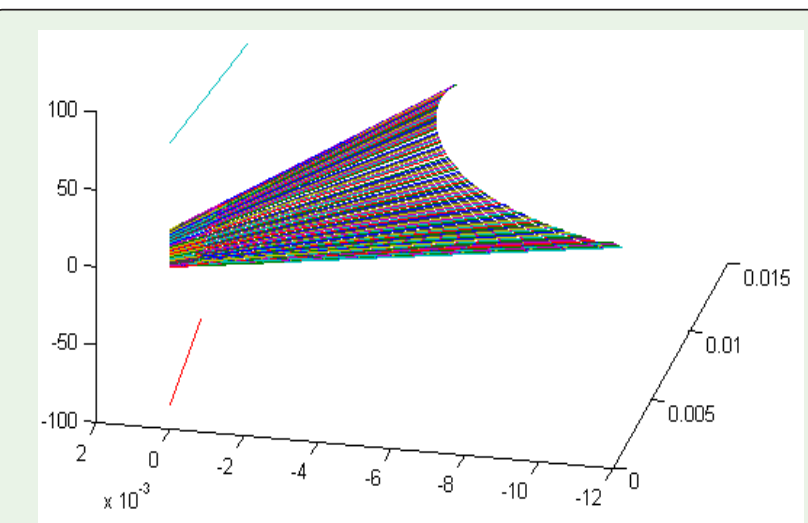

Figure 1: The tensegrity structure of the shank and thigh forms two levels of hierarchy at the knee.

To obtain values of these ratios at a special configuration having torqueless connections, i.e., knee tensegrity system [7], we need to specify first that the IAK about which the knee is required to twist with finite amplitude and the GRF on which the foot will deliver a force of finite intensity, are covariant during locomotion. Thus, the knee tensegrity system guarantees both sensitivity $1=\infty$ and sensitivity $2=0$. Additionally, we can also define the haptic control rules as

Tactile scanning = variation in motor activity/Variation in skin stimulation

Articular scanning $=($ variation in muscular activity $) /($ variation in joint stimulation)

To validate the proposed sensitivity of touch to knee kinesthesis during the stance phase of gait, we used previously published experimental datasets and had measured the KIA through readily accessible benchmark data [8]. Also, we have measured the GRF on how the progression of the entire body over the limb uses so-called 'rockers' on foot.

\section{Results and Discussion}

Any change in the pattern of contacts with the skin is covariant and concomitant with a change in the branching of bones. The touch pattern and the vector pattern are altered together by the mechanical necessities of terrestrial movement-the "sense of support (Figure 2)". The covariance of cutaneous and articular motion is information. The neural input with covariation is different from either of the two inputs without covariation.

A motor act normally yields both skin kinesthesis and joint kinesthesis. Together, they have a different meaning than would a simple movement of the skin or a simple movement of the bones. And an invariant unity between them specifies an external layout of surfaces. If a terrestrial surface is nearly horizontal (instead of slanted), nearly flat (instead of convex or concave) and sufficiently extended (relative to the size of the animal) and if its substance is rigid (relative to the weight of the animal), then the surface affords support [1].

A unique combination of invariants, a compound invariant, is just another invariant. It is a unit, and the components do not have to be combined or associated. Only if percepts were combinations of sensations would they have to be associated. Otherwise, we can

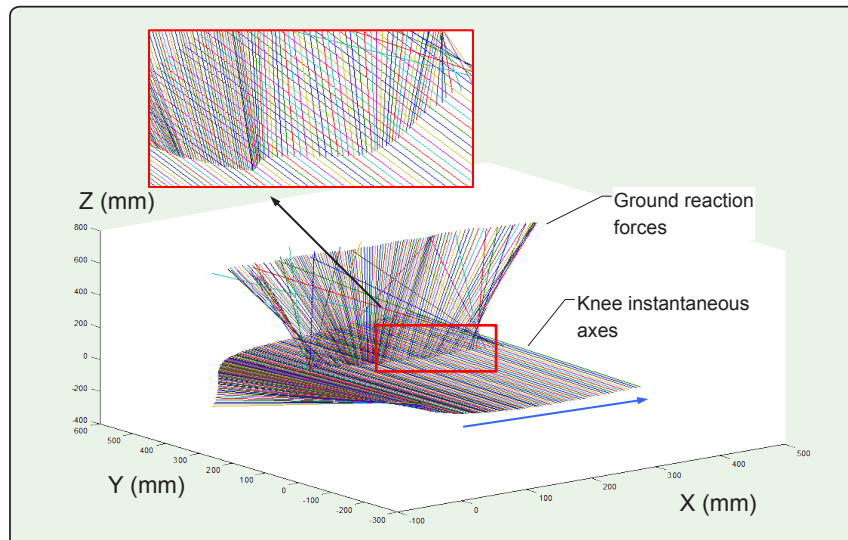

Figure 2: A unique combination of the KIA and GRF stimuli.When deformed by the shank to the ground via GRF, the strain is distributed over the whole structure, not localized in the area being deformed, i.e., the joint itself. A reaction torque is zero on the knee joint if the GRF line of action intersects the joint axis, or the configuration can exert a large force on the ground without overloading the knee joint. A considerable ground reaction force can be exerted on a foot when the vector nearly coincides with a reciprocal screw of joints. It is indicative of the "sense of support" being manifested based on the close correspondence of the vector of the ground reaction force at COP, and the IAK with fluctuations at the spatial scale of a millimeter (GRF-KIA coupling).

postulate that when the KIA and GRF are completely covariant when they always go together, they constitute a single "stimulus." If the knee tensegrity system [7] is capable of extracting invariants from a changing haptic stimulus, there is no reason why it should not extract invariants that seem to us highly complex. Therefore, the reaction torque caused by the foot-ground at the knee will be taken on partially by muscles surrounding the joint.

We need to establish a new paradigm for braces and orthosis intended to reduce the load that promises a beneficial effect for musculoskeletal disorders. Is the aspect of unloading essential, or are stability and kinesthesis the most important factors? The question of the latter can be answered by the exploration of 'rules for the haptic control of locomotion.'

\section{References}

1. Gibson JJ. The ecological approach to visual perception, Houghton Mifflin 1979.

2. Kim W, Espanha M, Veloso A, Araújo D, João F. "An Informational Algorithm as the Basis for Perception-Action Control of the Instantaneous Axes of the Knee." J Nov Physiother. 2013; 3: 127.

3. Kim W, Choi Y, Lee HG. "The Duality of Knee Functional Axes and Foot Contact." Journal of Functional Morphology and Kinesiology. 2016; 1: 387.

4. Gibson JJ. The senses considered as perceptual systems. Boston, Houghton. 1966.

5. Dooner DB. "On the Three Laws of Gearing." Journal of Mechanical Design. 2002; 124: 733-744.

6. Ball R. A treatise on the theory of screws, Cambridge University Press. 1900.

7. Kim W, Park A. "Topological Space of the Knee Tensegrity System." Biomed J Sci \&Tech Re. 2018; 3.

8. Fregly BJ, Besier TF, Lloyd DG, Delp SL, Banks SA, Pandy MG. "Grand challenge competition to predict in vivo knee loads." Journal of Orthopaedic Research. 2012; 30: 503-513. 